\title{
Uma Contribuição ao Estudo do Processo TIG Cold Wire na Posição Plana, Horizontal e Sobrecabeça sob Novos Desenhos de Tocha e Metodologias de Alimentação de Arame
}

\author{
Víctor Vergara Díaz ${ }^{1}$, John Michea Cortes ${ }^{1}$, Abdón Espinosa Alvarez ${ }^{1}$ \\ 1 Laboratório de Procesos de Soldadura, Departamento de Ingeniería Mecánica, Universidad de Antofagasta, Campus Coloso, \\ Antofagasta, Chile.
}

Recebido: $16 \mathrm{Jul}, 2015$

Aceito: 05 Out, 2015

E-mail: victor.vergara@uantof.cl (VVD)
Resumo: Esta linha de pesquisa surge para melhorar o atual procedimento na soldagem de tubos de caldeiras, onde são utilizados processos de soldagem manual e a qualidade das uniões soldadas depende exclusivamente da habilidade do soldador. Neste sentido, a proposta consiste em realizar a reparação com um processo de soldagem mais produtivo denominado de TIG cold wire com alimentação mecanizada de arame frio com a intenção de conseguir ganhos em qualidade nas uniões soldadas e repetitividade. Os cordões de solda foram avaliados pelo aspecto superficial, morfologia, grau de diluição e índice de convexidade. Foram realizados ensaios com dois direcionadores de arame $\left(20^{\circ}\right.$ e $\left.48^{\circ}\right)$ para verificar a influência do ângulo de entrada da injeção de arame no arco. Os resultados indicam um aumento considerável da produtividade do processo TIG com alimentação mecanizada de arame, quando é empregado o direcionador desenvolvido com entrada de arame de $20^{\circ}$, aproximando-se a taxa de deposição daquelas obtidas com o processo MIG/MAG, para um mesmo diâmetro de arame. Foi possível testar duas configurações de alimentação de arame: contínua e pulsada. Os resultados mostraram que a configuração de alimentação pulsada de arame proposta foi aplicada com sucesso em todas as posições garantindo repetitividade, estabilidade do processo e sustentabilidade da poça de fusão.

Palavras- chave: Posição de soldagem; Processo de soldagem TIG; Oscilação mecânica do arco.

\section{A Contribution to the Study of Cold Wire TIG Process in Flat, Vertical and Overhead Positions under New Torch Designs and Wire Feed Methodologies}

\begin{abstract}
This line of research aims to improve the current boiler tube welding procedure, where processes for manual welding are used and the quality of the welded joints depends exclusively on the skill of the welder. In this regard, the proposal aims to carry out the reparation using a more productive welding process called cold wire TIG, with mechanical cold wire feeding, with the intention of getting better quality and repeatability in the welded joints. We evaluated the surface appearance, morphology, degree of dilution and convexity rate of the weld beads. Assays were performed with two wire guide manipulators $\left(20^{\circ}\right.$ and $\left.48^{\circ}\right)$ to verify the influence of the entry angle of the wire injection at the arc. The results indicated that there is a considerable increase in TIG productivity with mechanical wire feeding when the guide manipulator with a wire entry of $20^{\circ}$ is used, approaching the deposition rate obtained with the MIG/MAG welding process for the same wire diameter. It was possible to test two wire feed settings: continuous and pulsed. These results show that the proposed configuration of pulsed wire feeding was successfully applied in all positions ensuring repeatability, process stability and sustenance of the weld pool.
\end{abstract}

Key-words: Welding positions; Welding process TIG; Mechanical oscillation arc.

\section{Introdução}

A motivação para realizar o presente estudo surgiu durante um trabalho realizado no Centro de Engenharia e Tecnologia dos Materiais da Universidade de Antofagasta que consistiu em fazer uma analises de falhas a tubos de caldeiras. $O$ estudo levou à conclusão que a origem da falha nos tubos de caldeiras foi pela incorreta aplicação dos cordões de solda e pela falta de um procedimento de soldagem adequado [1]. Tradicionalmente o procedimento consiste em fazer um passe de raiz realizado de forma manual pelo processo de soldagem TIG, sendo o preenchimento da junta, realizado pelo processo de soldagem a arco com eletrodo revestido SMAW. 
Foi realizada uma proposta para melhorar o atual procedimento de soldagem. A proposta consiste em utilizar o processo de soldagem TIG cold wire com alimentação mecanizada de arame e empregando-se a corrente contínua pulsada com frequências de $2,5 \mathrm{~Hz}$ e $5 \mathrm{~Hz}$. Assim, mediante um único processo de soldagem é possível fazer o passe de raiz e em seguida preencher a junta com oscilação mecânica do arco [2,3]. Um programa de controle do processo de soldagem TIG cold wire foi desenvolvido pela IMC, especificamente para esta aplicação.

Diante do exposto, duas configurações de alimentação de arame foram propostas: Alimentação contínua de arame (Programação de uma mesma velocidade de arame para a fase de base e para a fase de pulso da corrente) e alimentação pulsada de arame (Programação de velocidades diferentes de arame na fase de base e na fase de pulso da corrente).

Neste trabalho foram considerado os resultados obtidos por Schwedersky et al. [4] que relatam que existe uma grande possibilidade de melhoria de produtividade do processo de soldagem TIG por meio da utilização de frações de $\mathrm{H}_{2}$ no gás de proteção, principalmente em aplicações automatizadas que permitem maiores velocidades de soldagem.

Para a realização do estudo foi necessário projetar um direcionador de arame com o objetivo de injetar com arame diretamente na poça fundida. $O$ direcionador foi projetado com um ângulo de $20^{\circ}$ em relação ao eletrodo de tungstênio não consumível [5], técnica conhecida na bibliografia especializada como alimentação tangencial [6]. A alimentação radial foi realizada mediante um direcionador comercial que permite a entrada de arame no arco com um ângulo de $48^{\circ}$.

\section{Metodologia Experimental}

A Figura 1 mostra a bancada de soldagem montada no Laboratório de Processos de Soldagem (LPS) composta basicamente por uma fonte de Soldagem MINITEC 200, tocha de Soldagem TIG adaptada que permite a injeção de arame de forma radial ou tangencial, sistema de deslocamento da tocha com dois graus de liberdade, gases e Sistema de Aquisição de Dados (SAD).

Foram realizados cordões com simples deposição na posição plana, horizontal e sobrecabeça sobre chapas de aço SAE 1016 de dimensões de 200×63×100 mm. A Figura 2 mostra as três posições de soldagem testadas. Os fatores de controle avaliados foram: Ângulo de alimentação do metal de adição $\left(48^{\circ}\right.$ e $\left.20^{\circ}\right)$, posição de soldagem (Plana, Horizontal e Sobrecabeça), modo de alimentação de arame (Constante e pulsado), ângulo de inclinação da tocha de soldagem ( $\alpha$ : Posição horizontal; $\beta$ : Posição sobrecabeça), velocidade de soldagem (Vs).

Os parâmetros que se mantiveram constante durante a execução dos ensaios foram: A distância eletrodo-peça de $5 \mathrm{~mm}$, a distância da ponta do eletrodo em relação à face externa do bocal de gás de proteção foi de $5 \mathrm{~mm}$, ângulo de entrada de arame no arco de $20^{\circ}$ medido em relação ao eletrodo de tungstênio, material de aporte

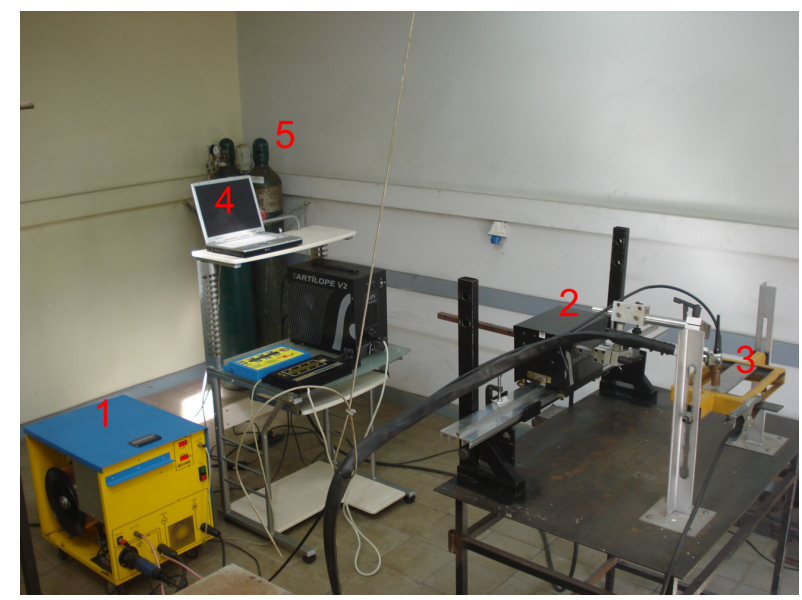

Figura 1. Bancada de soldagem para o processo de soldagem TIG com adição mecanizada de arame. 1) Fonte de soldagem. 2) Sistema de deslocamento com oscilação da tocha de soldagem. 3) Tocha TIG adaptada. 4) Sistema de aquisição de dados. 5) Gases de proteção. 

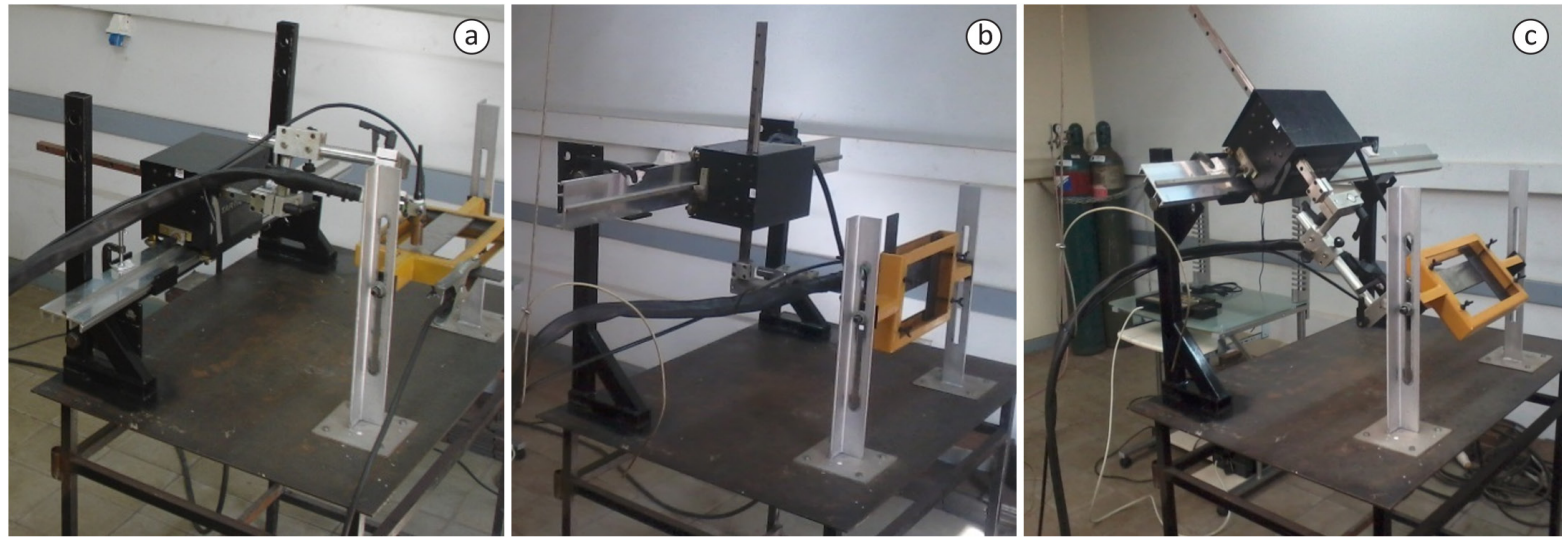

Figura 2. Bancada de ensaios com oscilação mecânica simulando diferentes posições de soldagem: (a) Plana. (b) Horizontal. (c) Sobrecabeça.

ER70S-6 de 0,8 mm de diâmetro, gás de proteção $\mathrm{Ar}+2 \% \mathrm{H}_{2}$ com uma vazão de $12 \mathrm{l} / \mathrm{min}$, eletrodo não consumível EWTh-2 de 2,4 mm diâmetro com um ângulo de afiação na ponta de $30^{\circ}$. A mistura de gás de proteção selecionada foi baseada nos resultados de Schwedersky et al. [4] que relatam que o uso de misturas $\mathrm{Ar}_{-} \mathrm{H}_{2}$ no gás de proteção se apresenta como uma alternativa para aumentar a velocidade de soldagem no processo TIG. Inicialmente os corpos de prova foram submetidos a inspeção visual quanto a presença de defeitos de soldagem, foram medidos os parâmetros geométricos do cordão de solda, índice de convexidade e o grau de diluição em uma macrografia da seção transversal dos depósitos, ataque com nital 2\%. O grau de diluição foi determinado pelo método das áreas.

Foi necessário, nas posições horizontal e sobrecabeça, inclinar a tocha TIG com um ângulo em relação ao eixo perpendicular ao substrato com o intuito de suportar a poça de fusão com a força do arco e diminuir desta forma o efeito de aparecimento das mordeduras nos cordões de solda [7-9]. A Figura 3 mostra o posicionamento do eletrodo de acordo à posição de soldagem.

\section{Resultados e Discussão}

\subsection{Desempenho do direcionador de arame}

Dois direcionadores de arame foram testados neste estudo os que foram denominados de direcionador com injeção radial de arame e direcionador com entrada tangencial de arame [6]. A Figura 4a mostra o direcionador radial de arame com um ângulo de injeção de $48^{\circ}$. A Figura $4 \mathrm{~b}$ mostra o direcionador de arame tangencial com um ângulo de injeção de $20^{\circ}$.
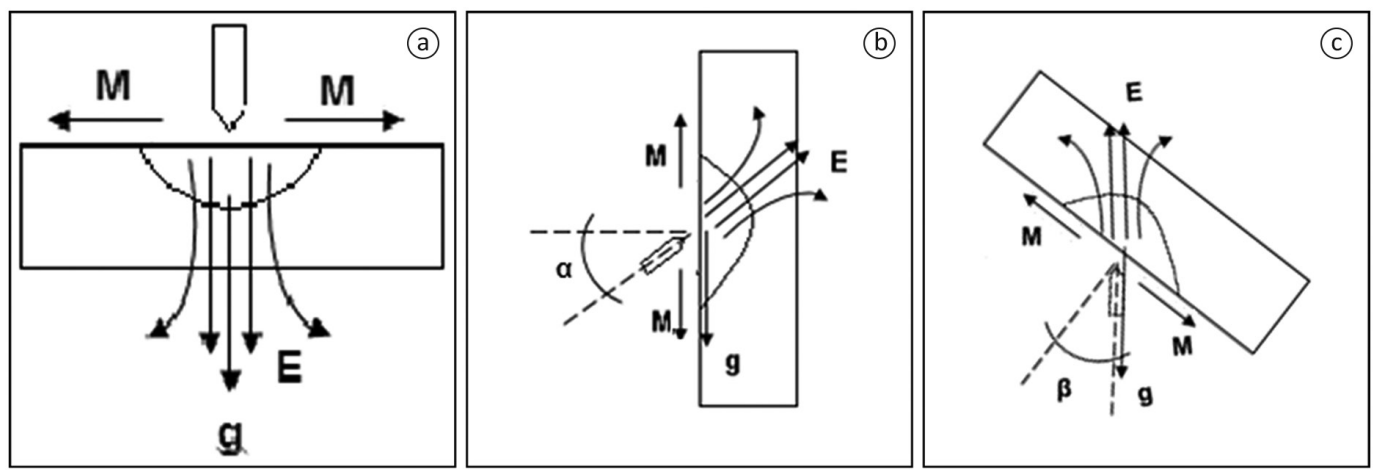

Figura 3. Esquema das forcas na formação da poça de fusão e posicionamento do eletrodo de acordo à posição de soldagem: (a) Plana. (b) Horizontal. (c) Sobrecabeça. E: Efeito eletromagnético. M: Convecção Marangoni. g: Forca de gravidade. $\alpha$ : Ângulo de inclinação do eletrodo não consumível na posição de soldagem horizontal. $\beta$ : Ângulo de inclinação do eletrodo na posição de soldagem sobrecabeça. 

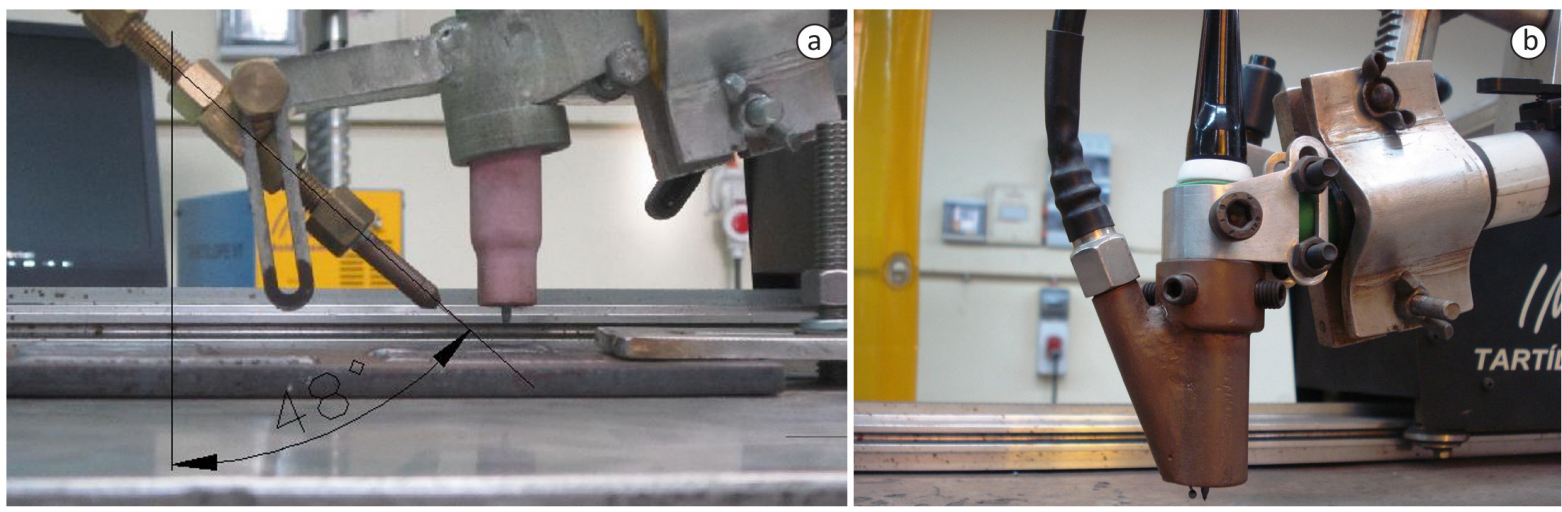

Figura 4. Direcionadores de arame. (a) Injeção radial de arame. (b) Injeção tangencial de arame.

Das duas técnicas testadas a que apresentou melhor desempenho em relação à taxa de deposição foi o direcionador com injeção de arame de forma tangencial (entrada de $20^{\circ}$ ) conseguindo-se valores de velocidade de arame de $4 \mathrm{~m} / \mathrm{min}(0,92 \mathrm{~kg} / \mathrm{h})$, valores de taxa de deposição bem próximo aos conseguidos no processo de soldagem MIG/MAG, quando se utiliza uma velocidade de arame de $5 \mathrm{~m} / \mathrm{min}(1,1 \mathrm{~kg} / \mathrm{h})$ com transferência metálica por curto circuito (Figura 5). Para velocidades de arame programados acima de $4 \mathrm{~m} / \mathrm{min}$ os cordões de solda ficaram com um reforço excessivo comprometendo a qualidade do cordão. $O$ valor máximo da velocidade de arame conseguido para o direcionador de arame radial foi de $2 \mathrm{~m} / \mathrm{min}$, acima deste valor o arame impacta o substrato devido à energia do arco não ser suficiente para fundir o arame.

\subsection{Configurações de corrente e velocidade de arame}

Neste trabalho serão estudadas duas configurações de alimentação de arame: Uma configuração que permita realizar cordões de solda com corrente contínua pulsada e com alimentação constante de arame (Figura 6a). A segunda proposta é produzir cordões de solda com corrente contínua pulsada e alimentação de arame pulsado, ou seja, alimentar na fase de base da corrente com uma velocidade de arame (Vab) e com outra velocidade de arame alimentar na fase de pulso da corrente (Vap). A Figura 6b mostra a segunda configuração proposta.

\subsection{Ensaios na posição plana}

As Tabelas 1 e 2 mostram o planejamento experimental. A Figura 7 apresenta o aspecto superficial dos cordões de solda realizados com alimentação de arame pulsada. Os ensaios foram realizados com oscilação mecânica do arco variando a velocidade de arame na base e no pulso. $O$ ensaio $10 \mathrm{P}$ foi realizado com uma velocidade de arame na base vab=0,5 m/min resultando num cordão com aspecto irregular devido ao baixo valor de vab. $O$ arame

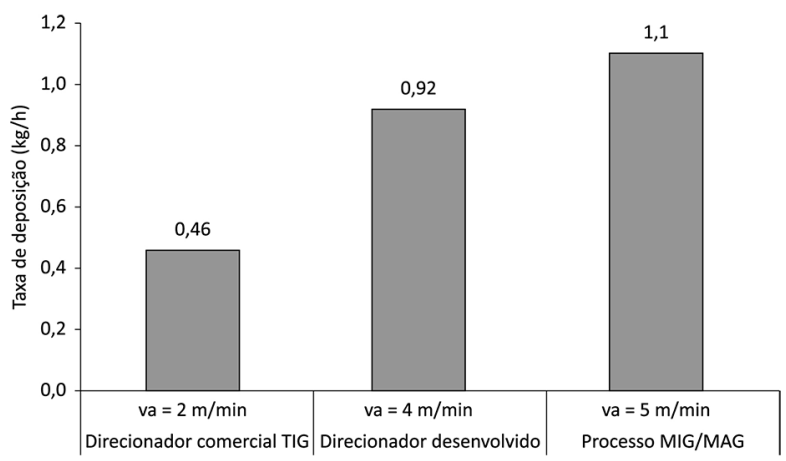

Figura 5. Desempenho do direcionador de arame. Condições de soldagem MIG/MAG: Tensão de referência $(U r)=15 \mathrm{~V}$. Velocidade de arame $=5 \mathrm{~m} / \mathrm{min}$. Indutância $(K s=K d=5)$. Velocidade de soldagem $=12 \mathrm{~cm} / \mathrm{min}$. Distância do bico de contato à peça $(D B P)=12 \mathrm{~mm}$. Vazão de gás de proteção $=16 \mathrm{l} / \mathrm{min}$. Tipo de gás de proteção $=80 \%$ $\mathrm{Ar}+20 \% \mathrm{CO}_{2}$. 
Uma Contribuição ao Estudo do Processo TIG Cold Wire na Posição Plana, Horizontal e Sobrecabeça sob Novos Desenhos de Tocha e Metodologias de Alimentação de Arame

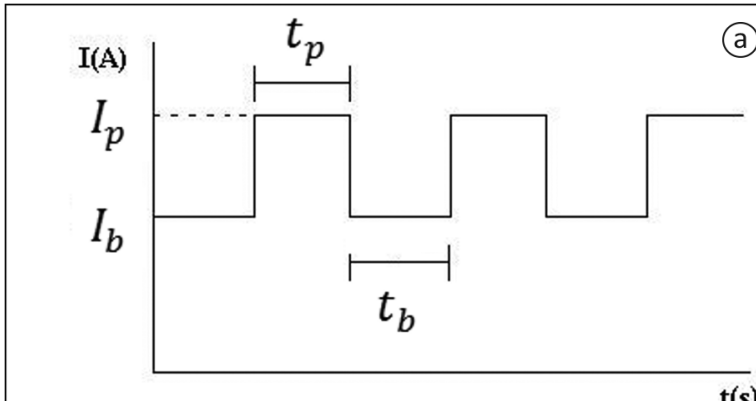

va(m/min)

$V_{a}$

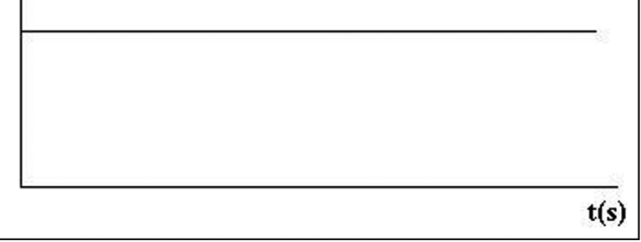

(a)

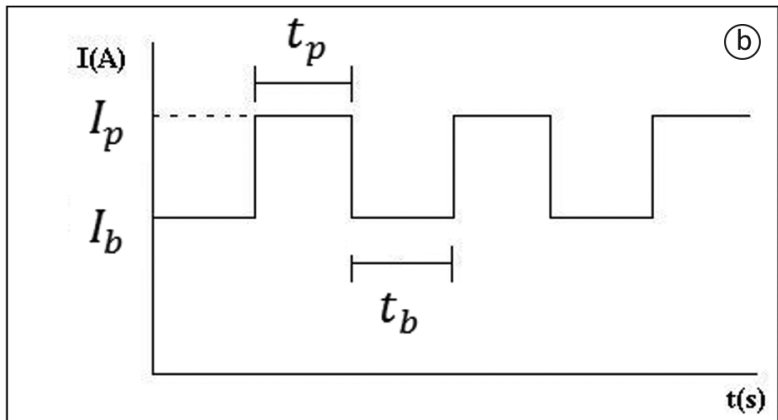

va(mimin)

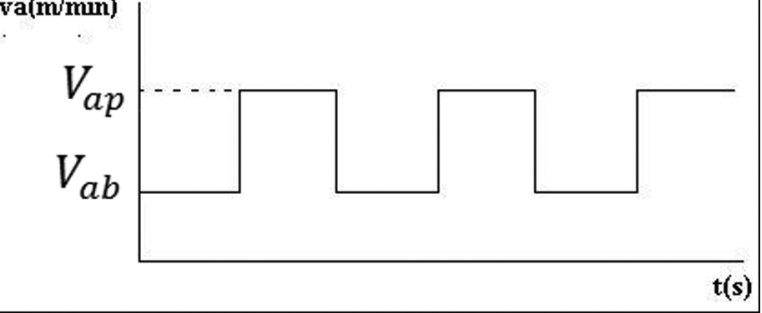

Figura 6. Esquema da corrente pulsada com (a) alimentação de arame constante, (b) arame pulsado. $l_{p}$ : Corrente de pulso. $I_{b}$ : Corrente de base. $t_{p}:$ Tempo de pulso. $t_{b}:$ Tempo de base. $V_{a p}:$ Velocidade de arame no pulso. $V_{a b}:$ Velocidade de arame na base. $V_{a}$ : Velocidade de arame constante.

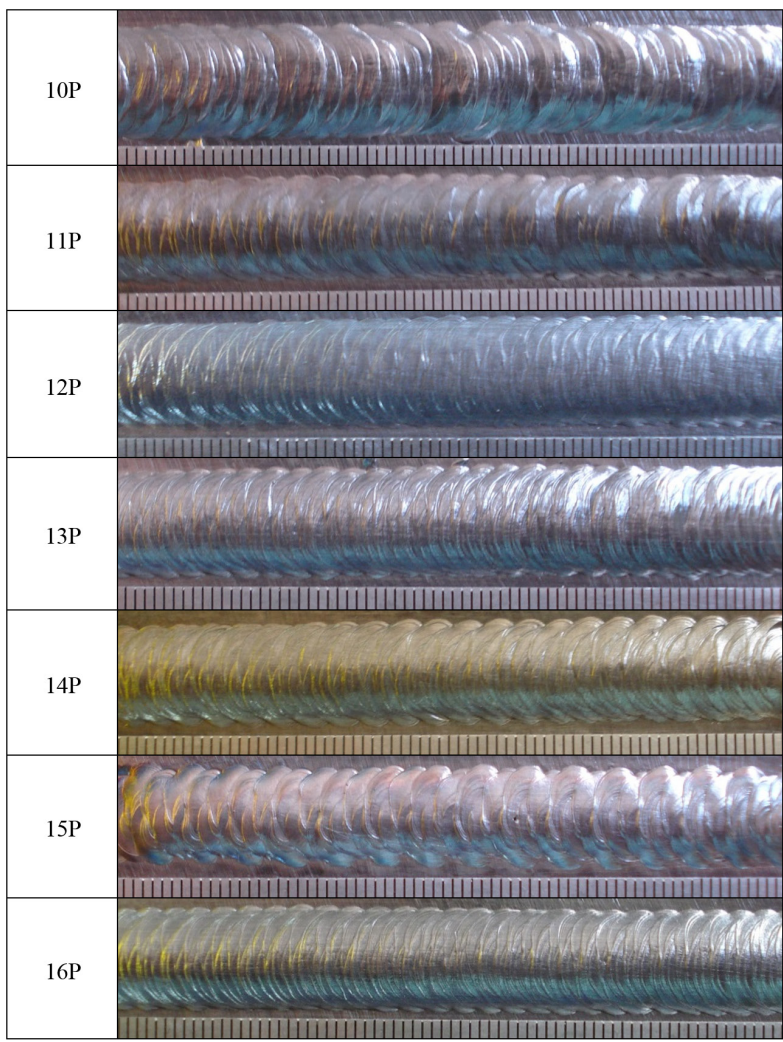

Figura 7. Aspecto superficial dos cordões realizados com alimentação de arame pulsada na posição de soldagem plana. 
Tabela 1. Parâmetros constantes para a configuração de alimentação de arame pulsada.

\begin{tabular}{cccccccccccc}
\hline$I_{p}(\mathbf{A})$ & $\boldsymbol{I}_{\boldsymbol{b}}(\mathbf{A})$ & $\boldsymbol{I}_{\boldsymbol{0}}(\mathbf{m m})$ & $\begin{array}{c}\boldsymbol{Q} \\
(\mathbf{I} / \mathbf{m i n})\end{array}$ & $\begin{array}{c}\text { Tipo gás de } \\
\text { Proteção }\end{array}$ & $\begin{array}{c}\text { Tipo de } \\
\text { Oscilação }\end{array}$ & $\boldsymbol{t}_{\boldsymbol{p} \mathbf{1}}(\mathbf{s})$ & $\boldsymbol{t}_{\boldsymbol{p} \mathbf{2}}(\mathbf{s})$ & $\begin{array}{c}\boldsymbol{A} \\
(\mathbf{m m})\end{array}$ & $\begin{array}{c}\boldsymbol{f}_{\text {osc }} \\
(\mathbf{H z})\end{array}$ & $\boldsymbol{t}_{\boldsymbol{p}}(\mathbf{s})$ & $\boldsymbol{t}_{\boldsymbol{b}}(\mathbf{s})$ \\
160 & 50 & 5 & 12 & $\mathrm{Ar}+2 \% \mathrm{H}_{2}$ & Triangular & 0 & 0 & 8 & 1 & 0,2 & 0,2 \\
\hline
\end{tabular}

$I_{p}$ : Corrente de pulso. $I_{b}$ : Corrente de base. $I_{0}$ : Comprimento do arco. $Q:$ Vazão de gás de proteção. $t_{p 1}:$ Tempo de parada. $t_{p 2}:$ Tempo de parada

2. A: Amplitude da oscilação. $f_{\text {osc }}$ : Frequência de oscilação. $t_{p}$ : Tempo de pulso. $t_{b}$ : Tempo de base.

Tabela 2. Parâmetros de soldagem para a configuração de alimentação de arame pulsada.

\begin{tabular}{cccccccc}
\hline Ensayo & $\mathbf{1 0 P}$ & $\mathbf{1 1 P}$ & $\mathbf{1 2 P}$ & $\mathbf{1 3 P}$ & $\mathbf{1 4 P}$ & $\mathbf{1 5 P}$ & $\mathbf{1 6 P}$ \\
$V_{a p}(\mathrm{~m} / \mathrm{min})$ & 3,0 & 3,0 & 3,0 & 3,5 & 3,5 & 3,5 & 3,5 \\
$V_{a b}(\mathrm{~m} / \mathrm{min})$ & 0,5 & 1,0 & 1,5 & 1,5 & 1,5 & 1,5 & 2,0 \\
$V_{s}(\mathrm{~cm} / \mathrm{min})$ & 10 & 10 & 10 & 10 & 12 & 15 & 10 \\
\hline
\end{tabular}

$V_{a p}:$ Velocidade de arame no pulso. $V_{a b}:$ Velocidade de arame na base. $V_{s}:$ Velocidade de soldagem.

nesta fase não consegue manter-se no interior do arco. Quando foi aumentada a velocidade de arame na base de 0,5 a $1,0 \mathrm{~m} / \mathrm{min}$ o aspecto superficial do cordão melhorou apresentando-se um cordão com um aspecto geométrico mais regular (Ensaio 11P). Finalmente foi testada uma vab=1,5 m/min, observando-se que o arame fica no interior do arco e próximo da poça de fusão. $O$ cordão apresenta um bom aspecto superficial (Ensaio 12P).

Para valores de vab superiores a $2,0 \mathrm{~m} / \mathrm{min}$ o arame toca o substrato mergulhando sobre a poça de fusão e devido à oscilação mecânica do arco a ponta do arame produz um arraste sobre a superfície do substrato. Esse efeito de arraste produz uma deflexão no arame desviando-o a regiões de menor energia no arco.

De acordo com os resultados experimentais obtidos, variações na programação da velocidade de arame na fase de base (vab) causam mudanças na morfologia do cordão de solda. A Figura 7 mostra o aspecto superficial dos ensaios 10P, 11P e 12P para velocidades de arame de 0,5, 1,0 e 1,5 m/min, respectivamente. A principal função da velocidade de arame na base é de manter o arame no interior do arco e próximo da poça de fusão, esperando o inicio da fase de pulso na qual o arame adicionado (vap) será fundido devido à corrente de pulso.

A corrente de base deve ser programada num valor mínimo, apenas suficientes para garantir que não ocorra a extinção do arco, sem que este valor de corrente de base funda o arame e o material base.

Os ensaios 13P, 14P e 15P da Tabela 2 mostram os valores da velocidade de soldagem utilizada de 10,12 e $15 \mathrm{~cm} / \mathrm{min}$, respectivamente. A Figura 7 mostra o aspecto superficial dos cordões de solda realizados com tecimento mecânico onde é possível observar defeitos como falta de fusão nas extremidades do cordão causado pela elevada velocidade de soldagem $(15 \mathrm{~cm} / \mathrm{min})$.

\subsection{Ensaios na posição horizontal}

Quando é empregada a configuração da Figura 6a, se apresentam dificuldades para sustentar a poça de fusão pela ação da força de gravidade, que age no sentido de fazer a poça escorrer. Nesta etapa é utilizada somente a configuração de alimentação pulsada de arame, Figura 6b. As Tabelas 3 e 4 mostram o planejamento experimental. $\mathrm{Na}$ execução dos ensaios foram observadas mordeduras no pé do cordão de solda (região superior). A solução foi inclinar a tocha TIG com um ângulo $\alpha$ em relação ao eixo perpendicular ao substrato com o intuito de suportar a poça de fusão com a força do arco e diminuir o efeito de aparecimento das mordeduras. A Figura 8 mostra uma comparação das seções transversais dos cordões de solda realizados para diferentes ângulos de inclinação do eletrodo no consumível em relação ao substrato, onde é possível observar a ausência das mordeduras.

Tabela 3. Planejamento dos ensaios para a configuração de alimentação pulsada de arame na posição de soldagem horizontal.

\begin{tabular}{cccccccccccc}
\hline Ensaio & $\mathbf{1 H}$ & $\mathbf{2 H}$ & $\mathbf{3 H}$ & $\mathbf{4 H}$ & $\mathbf{5 H}$ & $\mathbf{6 H}$ & $\mathbf{7 H}$ & $\mathbf{8 H}$ & $\mathbf{9 H}$ & $\mathbf{1 0 H}$ & $\mathbf{1 1 H}$ \\
$\mathrm{V}_{a p}(\mathrm{~m} / \mathrm{min})$ & 1,8 & 2,0 & 2,2 & 2,4 & 2,6 & 2,8 & 3,0 & 1,8 & 2,2 & 3,0 & 3,0 \\
$\mathrm{a}\left({ }^{\circ}\right)$ & 0 & 0 & 0 & 0 & 0 & 0 & 0 & 20 & 20 & 20 & 25 \\
\hline
\end{tabular}

$V_{a p}:$ Velocidade de arame no pulso. $\alpha$ : Ângulo de inclinação do eletrodo não consumível na posição de soldagem horizontal. 
Uma Contribuição ao Estudo do Processo TIG Cold Wire na Posição Plana, Horizontal e Sobrecabeça sob Novos Desenhos de Tocha e Metodologias de Alimentação de Arame

Tabela 4. Parâmetros constantes para a configuração de alimentação pulsada de arame na posição de soldagem horizontal.

\begin{tabular}{|c|c|c|c|c|c|c|c|}
\hline$I_{p}(\mathrm{~A})$ & $I_{b}(A)$ & $t_{p}(s)$ & $t_{b}(s)$ & $V_{a b}(\mathrm{~m} / \mathrm{min})$ & $V_{s}(\mathrm{~cm} / \mathrm{min})$ & $I_{0}(\mathrm{~mm})$ & $Q(1 / \mathrm{min})$ \\
\hline 160 & 50 & 0,2 & 0,2 & 0,5 & 10 & 5 & 12 \\
\hline$t_{p 1}(s)$ & 0 & $f_{\text {osc }}(H z)$ & 1 & $\begin{array}{c}\text { Tipo gás de } \\
\text { proteção }\end{array}$ & \multicolumn{3}{|c|}{ Argônio com 2\% de Hidrogênio } \\
\hline$t_{p 2}(s)$ & 0 & $A(\mathrm{~mm})$ & 8 & $\begin{array}{c}\text { Tipo de } \\
\text { oscilação }\end{array}$ & \multicolumn{3}{|c|}{ Triangular } \\
\hline
\end{tabular}

$I_{p}$ : Corrente de pulso. $I_{b}$ : Corrente de base. $t_{p}$ : Tempo de pulso. $t_{b}:$ Tempo de base. $V_{a b}$ : Velocidade de arame na base. $V_{s}:$ Velocidade de soldagem. $I_{0}$ : Comprimento de arco. $Q$ : Vazão de gás de proteção. $t_{p 1}$ : Tempo de parada. $t_{p 2}:$ Tempo de parada $2 . f_{o s c}$ : Frequência de oscilação. A: Amplitude da oscilação.
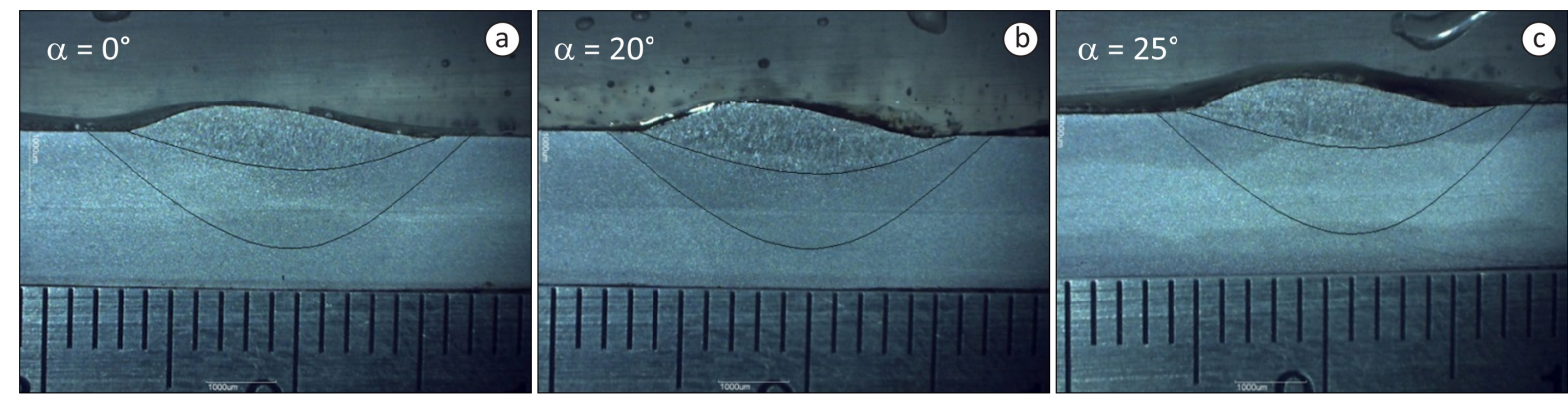

Figura 8. Seções transversais dos cordões realizados variando o ângulo de inclinação $(\alpha)$ do eletrodo não consumível na posição de soldagem horizontal. (a) $\alpha=0^{\circ}$ (Mordeduras). (b) $\alpha=20^{\circ}$ (Mordeduras). (c) $\alpha=25^{\circ}$ (Ausência de mordeduras).

\subsection{Ensaios na posição sobrecabeça}

Quando é empregada a configuração 6a, surgiram algumas dificuldades durante a execução dos ensaios. Devido à alimentação contínua de arame no arco, torna-se necessário programar variáveis de corrente de pulso e de base adequados para fundir o arame e manter um arco estável. Para conseguir boa estabilidade do arco foi necessário programar uma corrente de pulso de $160 \mathrm{~A}$ e a corrente de base foi variada em dois níveis: 100 e $120 \mathrm{~A}$. Para valores de corrente de base de $100 \mathrm{~A}$ a gota se forma na periferia do arco produzindo-se respingos os quais caem no interior do bocal interrompendo o processo por contaminação do eletrodo e desvio de arco. Para uma corrente de base de $120 \mathrm{~A}$ o processo apresenta boa estabilidade, mas a constante adição de material na poça de fusão não favorece a sustentação da poça e apresenta-se tendência a escorrimento causada pela força de gravidade. Este fenômeno se mostra na Figura 9 e na Tabela 5 se indicam os parâmetros de soldagem que foram empregados.

Logo, se procede a executar a configuração de alimentação pulsada de arame esquematizada na Figura $6 b$. Na aplicação deste modo de alimentação, o propósito é fundir o arame somente na fase de pulso com uma corrente de $160 \mathrm{~A}$, formando uma poça de fusão que possa sustentar-se para uma dada condição de velocidade de arame no pulso (vap). A corrente de base foi ajustada num valor de $50 \mathrm{~A}$, o suficiente para garantir que não ocorra a extinção do arco e permitir a aproximação da ponta do arame até a poça de fusão mediante a programação adequada da velocidade de arame na base (vab). $O$ aspecto superficial dos cordões de solda são apresentados na Figura 10 e a Tabela 6 mostra os parâmetros de soldagem empregados. Foi necessário realizar um ajuste da velocidade de arame na base e um ajuste na inclinação do ângulo $\beta$ da pistola em relação ao substrato. Quando foi utilizada uma velocidade de arame na base de $0,8 \mathrm{~m} / \mathrm{min}$ o arame impacta com o substrato desviando o arame a regiões de diferente energia no arco e afetando no aspecto superficial do cordão (Ensaio $12 \mathrm{SC}$, Figura 10). No ensaio $15 \mathrm{SC}$ da Figura 10, foi utilizada uma velocidade de arame de $0,5 \mathrm{~m} / \mathrm{min}$ e um ângulo de inclinação da pistola de $\beta=13^{\circ}$. Para este valor de velocidade de arame na base se elimina o efeito de colisão do arame com o substrato, permitindo que o arame avance até a poça de fusão durante a fase de base (Ver Figura 11e-g) e seja fundido na fase de pulso (Ver Figura 11h). 


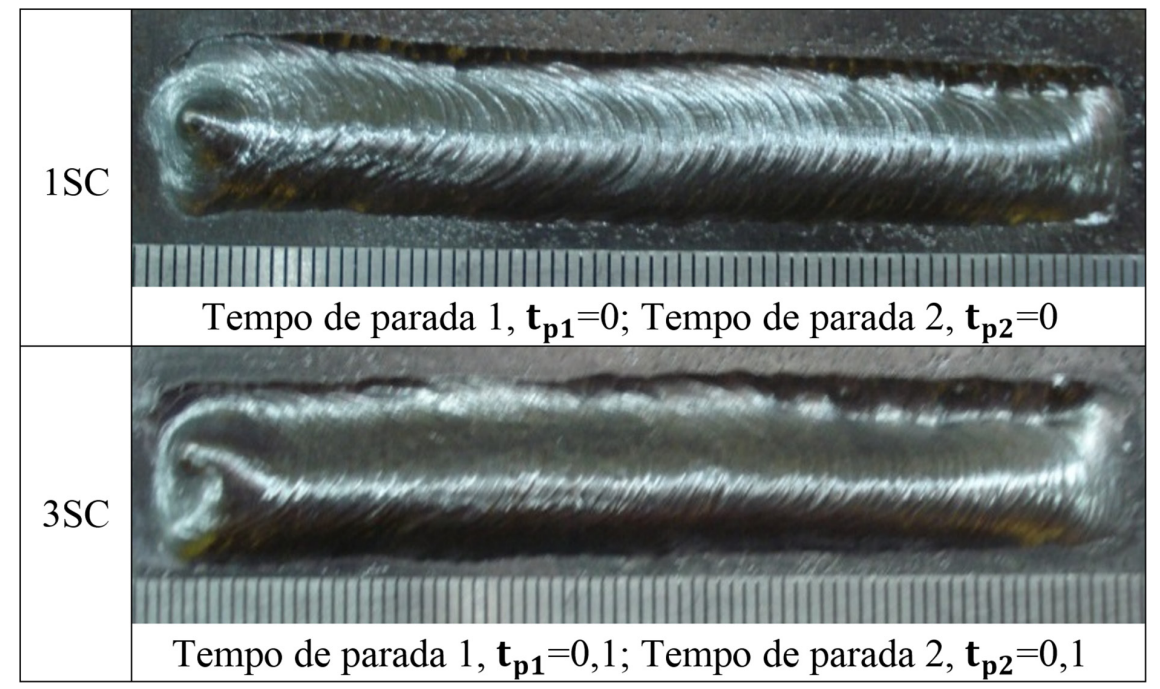

Figura 9. Aspecto superficial dos cordões de solda realizados na posição sobrecabeça com alimentação constante de arame.

Tabela 5. Parâmetros dos ensaios com alimentação constante de arame na posição de soldagem sobre-cabeça.

\begin{tabular}{ccccccccccc}
\hline$I_{p}(A)$ & $\boldsymbol{I}_{b}(\mathrm{~A})$ & $\boldsymbol{t}_{p}(\mathbf{s})$ & $\boldsymbol{t}_{\boldsymbol{b}}(\mathbf{s})$ & $\boldsymbol{V}_{\boldsymbol{a}}(\mathrm{m} / \mathrm{min})$ & $\boldsymbol{V}_{s}(\mathrm{~cm} / \mathrm{min})$ & $\boldsymbol{I}_{\boldsymbol{o}}(\mathrm{mm})$ & $\boldsymbol{Q}(\mathrm{I} / \mathrm{min})$ & $\boldsymbol{f}_{\text {osc }}(\mathrm{Hz})$ & $\boldsymbol{A}(\mathrm{mm})$ \\
160 & 120 & 0,1 & 0,1 & 2,0 & 10 & 5 & 12 & 1 & 8 \\
\hline
\end{tabular}

$I_{p}$ : Corrente de pulso. $I_{b}$ : Corrente de base. $t_{p}:$ Tempo de pulso. $t_{b}:$ Tempo de base. $V_{a}$ : Velocidade de arame constante. $V_{s}:$ Velocidade de soldagem. $I_{0}$ : Comprimento de arco. $Q$ : Vazão de gás de proteção. $f_{\text {osc }}$ : Frequência de oscilação. A: Amplitude da oscilação.

Tabela 6. Parâmetros dos ensaios com alimentação de arame de forma pulsada na posição de soldagem sobrecabeça.

\begin{tabular}{cccccccccc}
\hline$I_{p}(\mathbf{A})$ & $I_{b}(\mathbf{A})$ & $\begin{array}{c}\boldsymbol{t}_{p}=\boldsymbol{t}_{b} \\
(\mathbf{s})\end{array}$ & $\begin{array}{c}\boldsymbol{V}_{a p} \\
(\mathbf{m} / \mathbf{m i n})\end{array}$ & $\begin{array}{c}\boldsymbol{V}_{s} \\
(\mathbf{c m} / \mathbf{m i n})\end{array}$ & $\begin{array}{c}\boldsymbol{I}_{0} \\
(\mathbf{m m})\end{array}$ & $\begin{array}{c}\boldsymbol{Q} \\
(\mathbf{I} / \mathbf{m i n})\end{array}$ & $\begin{array}{c}\boldsymbol{f}_{\text {osc }} \\
(\mathbf{H z})\end{array}$ & $\begin{array}{c}\mathbf{A} \\
(\mathbf{m m})\end{array}$ & $\begin{array}{c}\boldsymbol{t}_{\boldsymbol{p} 1}=\boldsymbol{t}_{b 2} \\
(\mathbf{s})\end{array}$ \\
160 & 50 & 0,2 & 3,0 & 10 & 5 & 12 & 1 & 8 & 0 \\
\hline
\end{tabular}

Tipo gás de proteção: $\mathrm{Ar}+2 \% \mathrm{H}_{2}$. Tipo de oscilação: Triangular. $I$ : Corrente de pulso. $I_{t}$ : Corrente de base. $t$ : Tempo de pulso. $t_{t}$ : Tempo de base. $V_{a p}$ : Velocidade de arame no pulso. $V_{s}:$ Velocidade de soldagem. $I_{0}:$ Comprimento do arco. $Q$ : Vazão de gás de proteção. $f_{\text {osc }}:$ Frequência de oscilação. A: Amplitude da oscilação. $t_{p 1}$ : Tempo de parada 1. $t_{p 2}:$ Tempo de parada 2.

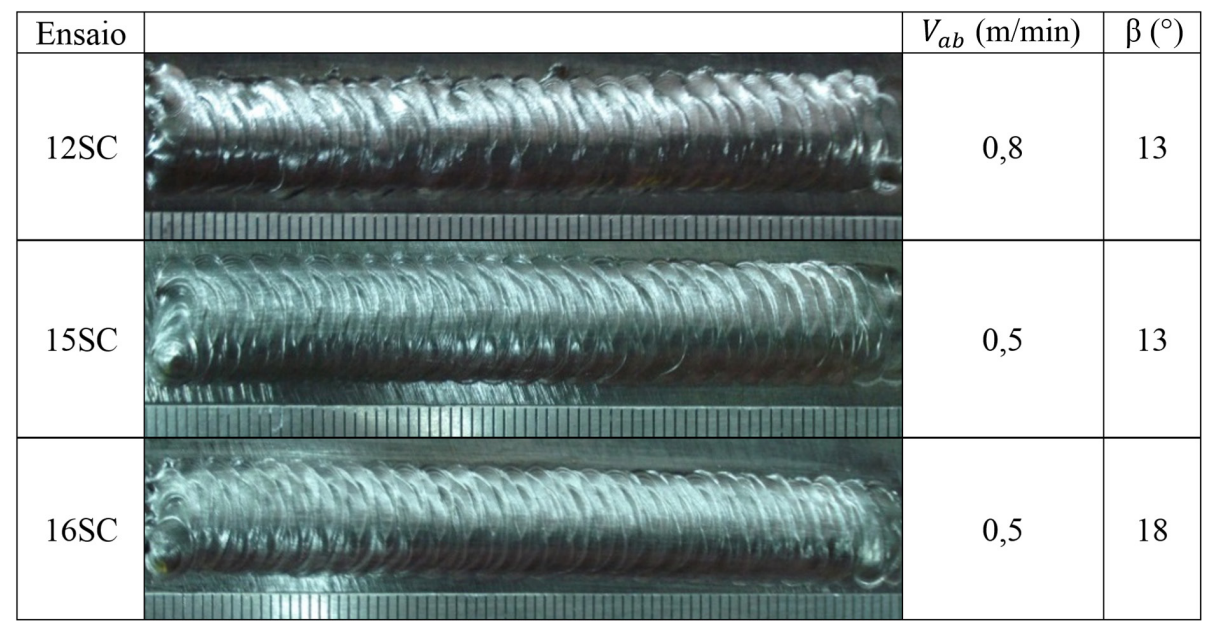

Figura 10. Aspecto superficial dos cordões obtidos variando a velocidade de alimentação de arame na base e ângulo de inclinação $(\beta)$ do eletrodo na posição de soldagem sobrecabeça. $V_{a b}$ : Velocidade de arame na base. $\beta$ : Ângulo de inclinação do eletrodo na posição de soldagem sobrecabeça. 


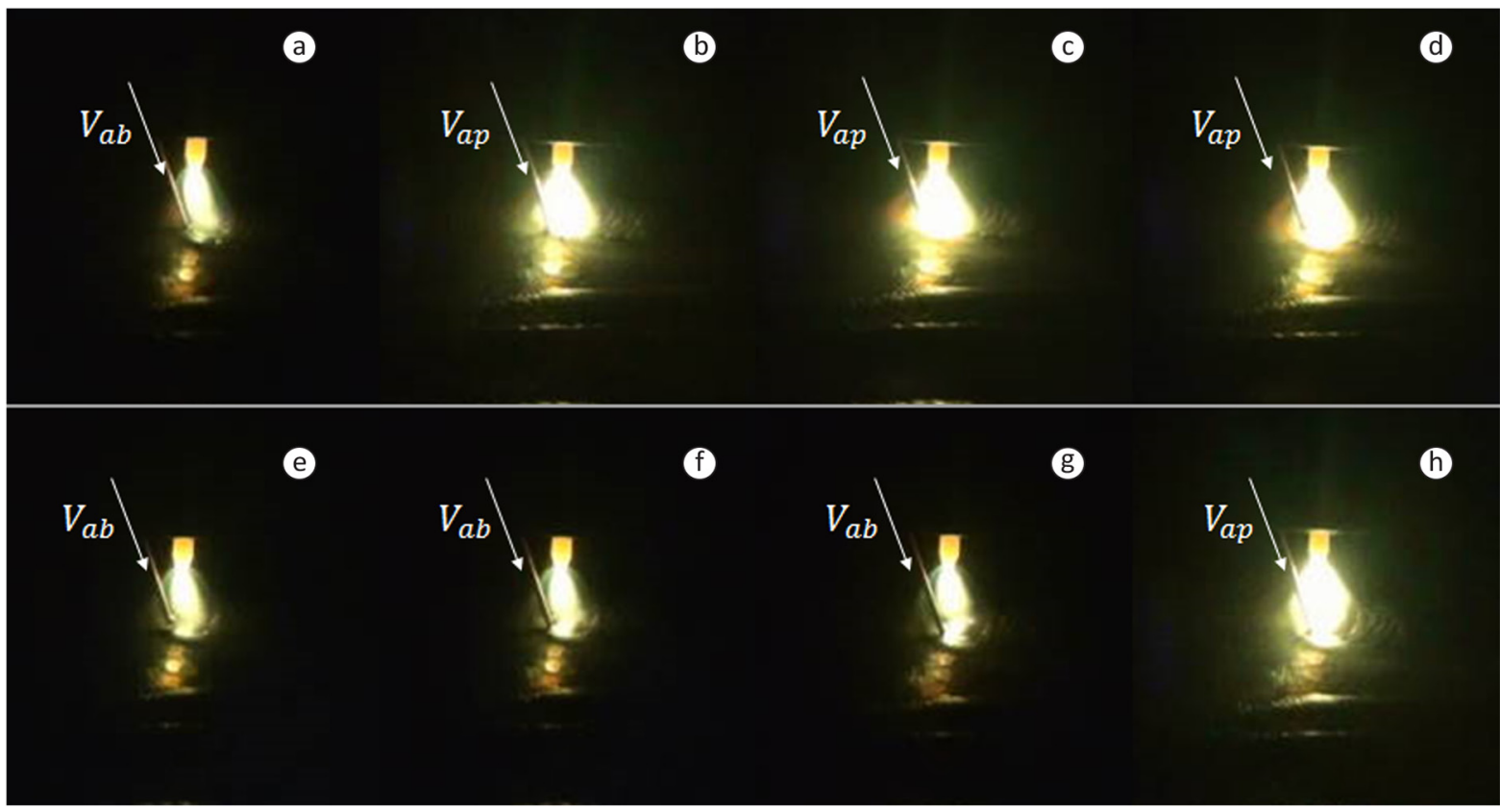

Figura 11. Sequência de imagens do arco evidenciando (a), (e), (f) e (g): Fase de base. (b), (c), (d) e (h): Fase de pulso. $V_{a b}$ : Velocidade de arame na base. $V_{a p}$ : Velocidade de arame no pulso.

Os melhores resultados foram conseguidos para uma velocidade de arame na base de $0,5 \mathrm{~m} / \mathrm{min}$ e com um ângulo de inclinação da pistola de $18^{\circ}$ (Ensaio 16SC, Figura 10). As mordeduras foram eliminadas quando o eletrodo no consumível foi inclinado um ângulo $\beta$ em relação ao substrato. As macrografias da Figura 12 revelaram cordões de solda isentas de mordeduras.

\subsection{Influência da posição de soldagem na geometria do cordão de solda}

A Figura 13 mostra a seção transversal dos cordões, onde é possível observar que o cordão realizado na posição plana (10P) apresenta uma diferença significativa na geometria da área depositada, em relação às outras posições de soldagem, $11 \mathrm{H}$ e $16 \mathrm{SC}$, devido a que nestas posições a poça fundida é afetada fortemente pelo efeito da gravidade.
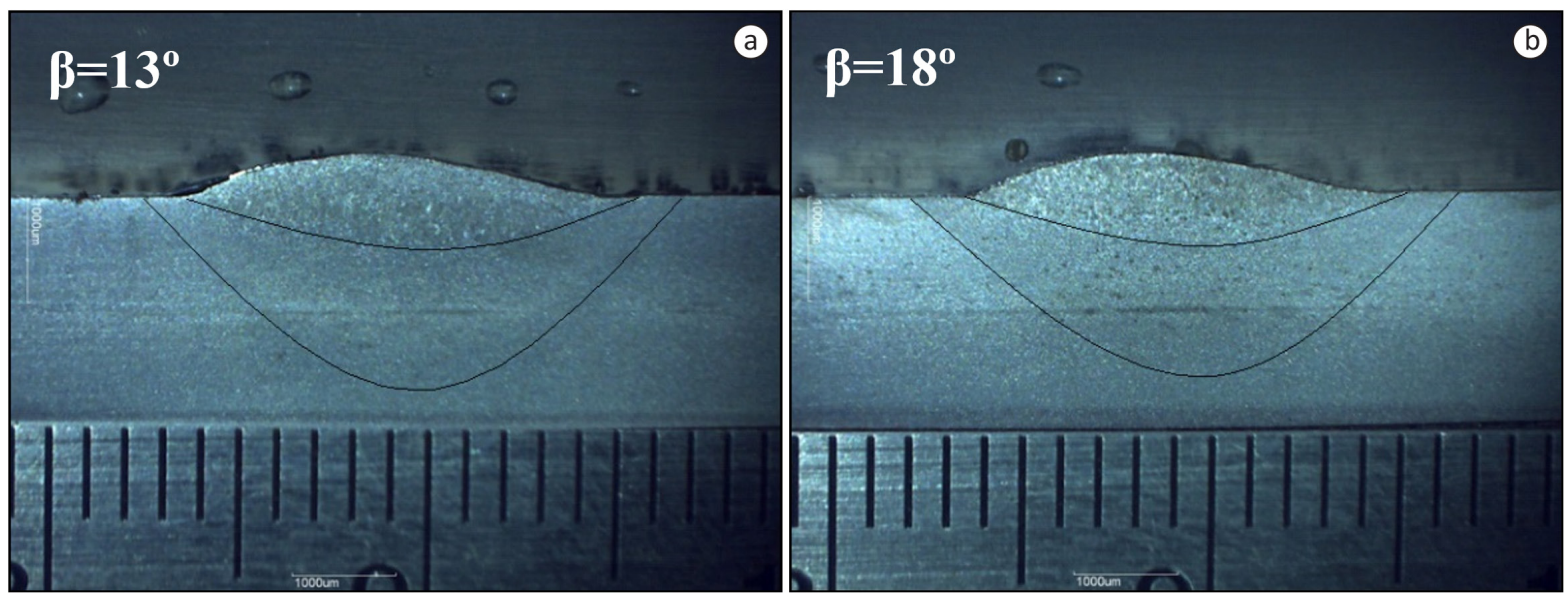

Figura 12. Macrografias dos ensaios realizados com variação do ângulo de inclinação ( $\beta$ ) do eletrodo na posição de soldagem sobrecabeça. (a) Ensaio 15SC, $\beta=13^{\circ}$. (b) Ensaio 16SC, $\beta=18^{\circ}$. 


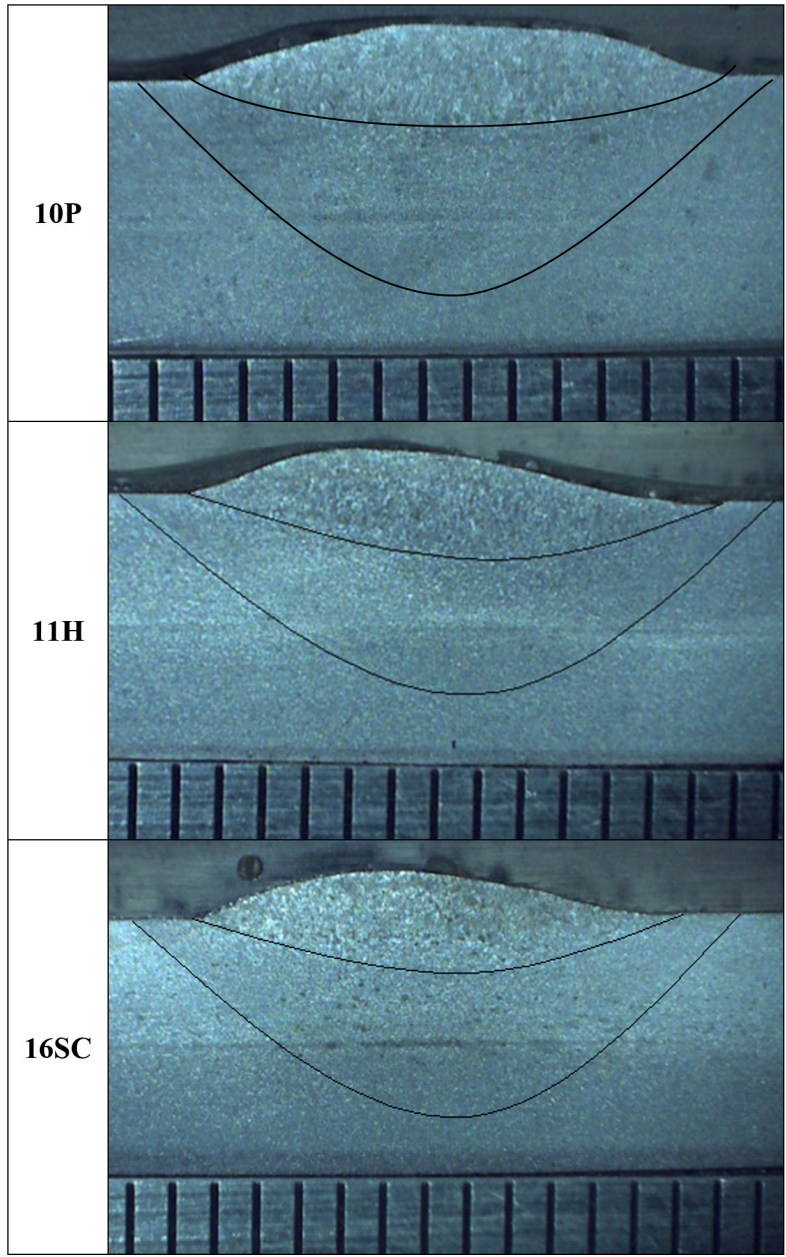

Figura 13. Seção transversal do cordão na posição plana (10P), horizontal (11H) e sobre sobrecabeça (16SC).

Tabela 7. Morfologia dos cordões realizados em similares condições nas posições de soldagem plana, horizontal e sobrecabeça.

\begin{tabular}{cccccccc}
\hline Ensaio & $\begin{array}{c}\mathbf{L} \\
\mathbf{( m m})\end{array}$ & $\begin{array}{c}\mathbf{R} \\
\mathbf{( m m})\end{array}$ & $\begin{array}{c}\mathbf{P} \\
\mathbf{( m m})\end{array}$ & $\begin{array}{c}\mathbf{A}_{\mathbf{d}} \\
\left(\mathbf{m m}^{\mathbf{2}}\right)\end{array}$ & $\begin{array}{c}\mathbf{A}_{\mathbf{f}} \\
\left(\mathbf{m m}^{2}\right)\end{array}$ & $\begin{array}{c}\delta \\
(\%)\end{array}$ & $\begin{array}{c}\text { IC } \\
(\%)\end{array}$ \\
$10 \mathrm{P}$ & 12,11 & 1,24 & 1,21 & 9,83 & 10,14 & 50,78 & 10,24 \\
$11 \mathrm{H}$ & 12,15 & 1,21 & 1,41 & 7,5 & 10,84 & 59,11 & 9,96 \\
$16 \mathrm{SC}$ & 11,84 & 1,11 & 1,49 & 7,34 & 10,83 & 59,60 & 9,38 \\
\hline
\end{tabular}

L: Largura. R: Reforço. P: Penetração. $A_{d}$ : Área depositada. $A_{f}$ : Área fundida. $\delta$ : Diluição. IC: Índice de convexidade.

A Tabela 7 apresenta os resultados sobre as condições geométricas, tais como largura, reforço, penetração, grau de diluição e índice de convexidade. As Figuras 14 e 15 indicam o efeito da posição de soldagem sobre as condições geométricas e a Figura 16 indica o efeito da posição de soldagem sobre o grau de diluição e índice de convexidade.

A análise da Figura 14 indica que a posição de soldagem afeta sobre a largura do cordão de solda. Na Figura 15 percebe-se um efeito significativo na penetração, apresentando-se os maiores valores na posição sobrecabeça e horizontal. Avaliando os resultados do reforço nota-se que os valores vão diminuindo da posição plana para a posição sobrecabeça. 
Uma Contribuição ao Estudo do Processo TIG Cold Wire na Posição Plana, Horizontal e Sobrecabeça sob Novos Desenhos de Tocha e Metodologias de Alimentação de Arame

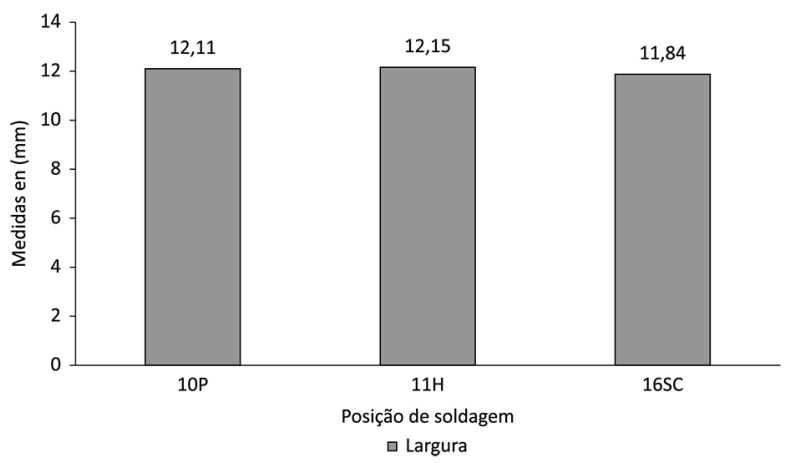

Figura 14. Largura dos cordões correspondentes aos ensaios 10P, 11H e 16SC.

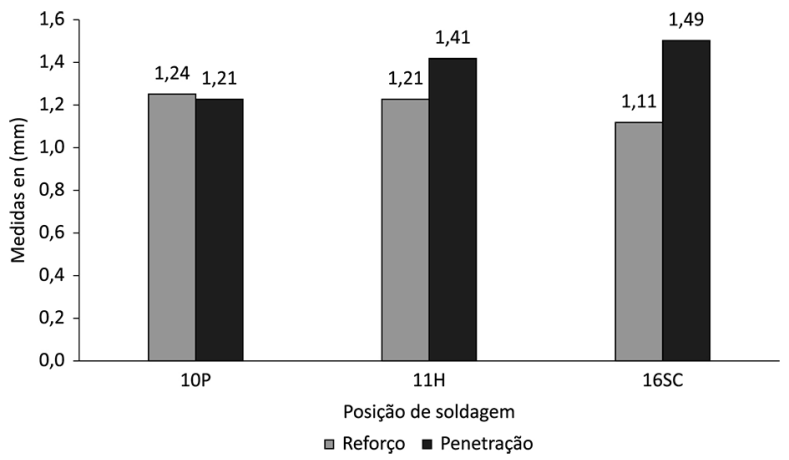

Figura 15. Reforço e penetração dos cordões correspondentes aos ensaios 10P, 11H e 16SC.

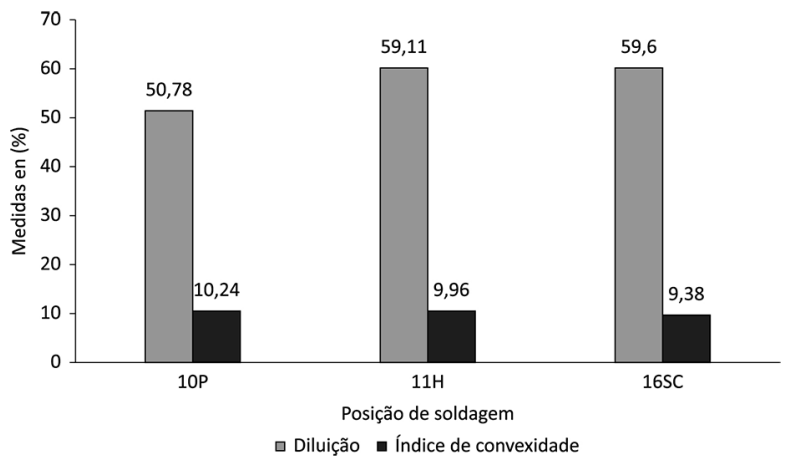

Figura 16. Diluição e índice de convexidade dos cordões correspondentes aos ensaios 10P, 11H e 16SC.

A análise da Figura 16 mostra que a posição de soldagem afeta sobre o grau de diluição e do índice de convexidade. $\mathrm{O}$ uso da técnica da oscilação mecânica do arco permitiu a obtenção de cordões de solda com baixos valores de índice de convexidade. Silva et al. [10] estabeleceram critérios de aceitabilidade sobre o índice de convexidade indicando que valores próximos de $15 \%$ representam uma condição de excelente qualidade. Para as posições de soldagem testadas o índice de convexidade sempre foi menor a 15\% (Figura 16), resultados que tornam-se mais úteis quando são utilizados em operações de revestimentos.

\section{Conclusões}

Com base nos resultados experimentais apresentados neste trabalho pode-se concluir que:

Com os parâmetros de soldagem estudados e ajustados, foi possível realizar cordões de solda de qualidade com oscilação mecânica do arco nas posições de soldagem plana, horizontal e sobrecabeça. 
O direcionador que apresentou melhor desempenho em relação à taxa de deposição foi o direcionador com injeção de arame de $20^{\circ}$, conseguindo-se valores de velocidade de arame de $4 \mathrm{~m} / \mathrm{min}$, valores de taxas de deposição, bem próximo aos conseguidos no processo de soldagem MIG/MAG.

Na configuração de alimentação de arame constante, a estabilidade do processo depende da correta seleção da corrente de base para evitar a formação da gota na periferia do arco o que pode gerar respingos e escorrimento de material. Porém, a configuração pulsada de alimentação de arame permite um excelente controle sobre a taxa de deposição e um correto posicionamento do arame no interior do arco voltaico. A configuração apresentou boa estabilidade no processo de soldagem podendo ser aplicada com sucesso a todas as posições de soldagem estudadas.

Nas posições horizontal e sobrecabeça foi necessário inclinar a tocha TIG em relação ao eixo perpendicular ao substrato com o intuito de suportar a poça de fusão com a força do arco e diminuir o efeito de aparecimento das mordeduras.

De acordo com os resultados experimentais obtidos, o nível de velocidade de arame programada na corrente de base tem como função manter o arame no interior do arco e próximo da poça de fusão.

O uso da técnica da oscilação mecânica do arco permitiu a obtenção de cordões de solda com baixos valores de índice de convexidade, resultados que tornam-se mais úteis quando são utilizados em operações de revestimentos.

\section{Agradecimentos}

Os autores apresentam seus agradecimentos, pela colaboração financeira a FIC-R 2012 e à Vicerrectoría de Investigación, Innovación y Postgrado da Universidade de Antofagasta através da bolsa Fondo de Investigación Desarrollo Tesis de Pregrado o Actividad de Titulación.

\section{Referências}

[1] Ochoa H, Henríquez R, Vergara V. Estudio de falla en intercambiador de calor. In: Resúmenes del 10을 Encuentro Internacional de Mantenedores de Plantas Mineras, MAPLA 2013; 2013 Sept 4-6; Santiago, Chile. Santiago: Gecamin; 2013. p. 123-124.

[2] Gonzalez E, Vergara V, Camus M, Espinosa A. Soldadura GTAW automatizada aplicada en uniones a tope en acero inoxidable 316L. In: Resúmenes del 9을 Encuentro Internacional de Mantenedores de Plantas Mineras; 2012 Sept 5-7; Santiago, Chile. Santiago: Gecamin; 2012.

[3] Schvartzman M, Fratari RQ, Scotti A. Otimização dos parâmetros de tecimento para confecção de amanteigamento em chapas de aço ao carbono pelo processo TIG com arame AWS ER309L. Soldagem \& Inspeção. 2010;15(3):209-217.

[4] Schwedersky MB, Dutra JC, Okuyama MP, Gonçalves e Silva RH. Soldagem TIG de elevada produtividade: influência dos gases de proteção na velocidade limite para formação de defeitos. Soldagem \& Inspeção. 2011;16(4):333-340.

[5] Fortain J, Rimano L, Vaidya V. Innovative process improves welding of sheet metal parts. Welding Journal. 2008;87(1):38-44.
[6] Silva RHG, Ribeiro JCB. Desenvolvimento de procedimentos de soldagem TIG e análise de diferentes parâmetros para a soldagem de raiz em tubos de aço API 5 L Grau A. In: Anais do XXXVII Congresso Nacional de Soldagem - CONSOLDA; 2011 Out 3-6; Natal, Brasil. Natal: ABS; 2011.

[7] Shirali A, Mills K. The effect of welding parameters on penetration in GTA Welds. Welding Journal. 1993;72:347-353.

[8] Cunha TV, Bohórquez CEN. Dispositivo para medição da pressão do arco e sua aplicação no estudo do processo TIG. Soldagem \& Inspeção. 2014;19(2):152-158. http://dx.doi.org/10.1590/01049224/SI1902.07.

[9] Savage WF, Nippes EF, Agusa K. Effect of arc force on defect formation in GTA welding. Welding Journal. 1979;(Supl):212s-224-s.

[10] Silva CR, Ferraresi VA, Scotti A. A quality and cost approach for welding process selection. Journal of the Brazilian Society of Mechanical Sciences. 2000;22(3):389-398. 